\title{
How chromosomes unite
}

The way in which chromosomes come together within a single nucleus after cell division has now been shown to involve a small DNA-binding protein named BAF, which might help to join chromosomes with one another.

\section{TEJAS DHARMARAJ \& KATHERINE L. WILSON}

$\mathrm{T}$ The basics of cell division are in some ways so familiar to many: make a copy of each chromosome, move these physically connected copies to the centre of the cell and separate the copies by pulling one member of each pair to opposite ends of the dividing cell, then package each set of chromosomes in an enclosing nuclear-envelope structure. This envelope consists of two concentric membranes (each a lipid bilayer) studded with various proteins and nuclear-pore structures that allow molecules to enter and leave the nucleus. However, many questions remain to be answered about the mechanisms underlying this cellular 'heartbeat' of life. Writing in Cell, Samwer et al. ${ }^{1}$ now shed light on the mystery of how chromosomes are packaged together in the same nucleus, an outcome known to help protect the integrity of the genome.

When a cell divides, as each chromosome is pulled away from the centre of the cell by ropelike strands of microtubule proteins, poised nearby are the disassembled and detached membrane and protein components that are needed to rebuild the nucleus. How can these elements capture all of the chromosomes within a single nucleus and avoid packaging chromosomes individually? Efficient capture is vital because lost chromosomes that stray too far from the chromosomal herd are packaged in separate micronuclei, which are fragile ${ }^{2}$. DNA in such micronuclei is prone to irreparable damage ${ }^{2}$ and micronucleus formation is associated with the generation of genetic mutations and cancer ${ }^{3}$. The fragile nature of micronuclei also means that the chromosomal DNA is often exposed to cytoplasmic proteins that can mistake it as a sign of infection, triggering an immune response and inflammation ${ }^{4}$.

Samwer and colleagues eliminated two possibilities for how chromosomal capture within the nucleus might occur. First, they tested whether microtubules are involved by adding a drug to cultured human cells that disassembles the microtubular strands. However, this treatment did not prevent the cells from forming a single nucleus. The researchers then tested whether chromosomes might coalesce because there is not enough nuclear-membrane
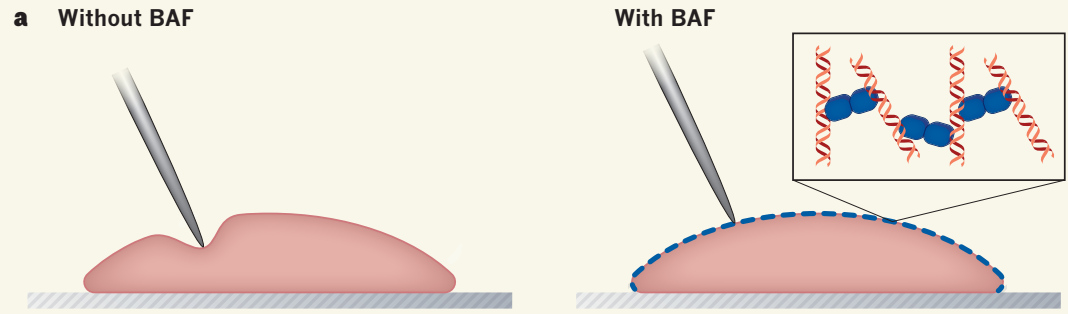

b
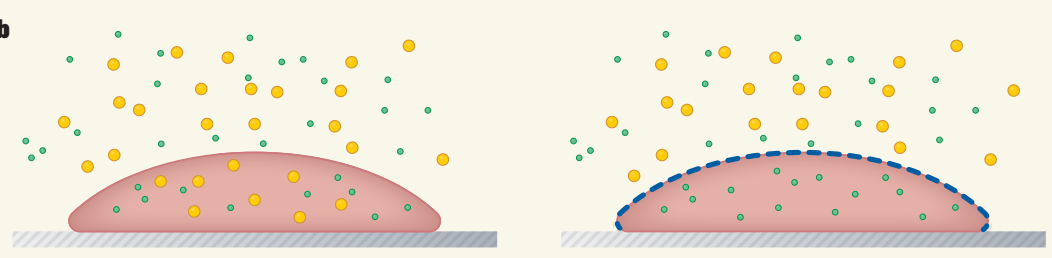

Figure 1 | BAF protein affects chromosomal structure. Samwer et al. ${ }^{1}$ investigated how groups of chromosomes coalesce within a single nucleus after cell division and found that BAF is essential to this process. a, The authors isolated purified chromosomes (pink) from cultured human cells, placed them on glass slides and incubated them in the presence or absence of purified BAF (blue). In the absence of BAF, chromosomes deformed when pressed with a metal rod, whereas in the presence of BAF they resisted deformation, suggesting mechanical stiffening. BAF dimers might stiffen chromosomes by linking adjacent DNA regions (inset). b. The authors observed that BAF makes chromosomes relatively impermeable to large (49-nanometre diameter) dextran molecules (yellow), whereas small (4-nm diameter) dextrans (green) can infiltrate the interior region of the chromosomal material. BAF might therefore also restrict the access of nuclear-membrane components that surround the nucleus (and have diameters of 60-100 nm), by limiting them to the outermost surfaces of grouped chromosomes.

material to surround each individual chromosome. But when cells were treated with a drug that kept chromosomes apart, a sealed nuclear envelope formed around nearly all of the individual chromosomes. Hence, nuclear membranes are not a limiting factor.

Undaunted, the authors tried something else. They systematically silenced, one at a time, the expression of 1,295 genes in human cells. These genes were selected because they are known to have a role in cell division. The consequences of losing each gene were studied using an algorithm that analysed thousands of images of cells, in which the nuclei were tagged with fluorescent markers to reveal their number and size.

The cells with the highest numbers of micronuclei lacked expression of the gene $B A N F 1$, which encodes a protein called BAF, an essential but surprisingly small protein comprising only 89 amino acid residues. How could this tiny protein control structures as large as chromosomes?

Previous studies ${ }^{5,6}$ of BAF offered possible clues. BAF is required, alongside nuclear-envelope proteins called lamins and LEM-domain proteins, to build a sealed, functional nuclear envelope after cell divi$\operatorname{sion}^{5,6}$. However, Samwer and colleagues discovered that a LEM-domain protein and the associated nuclear-envelope membranes were still recruited to chromosomes in BAFdepleted cells, and that these membranes now infiltrated the space between neighbouring chromosomes. When they tested cells containing a mutated version of BAF that couldn't bind LEM-domain proteins, they found that this mutant version worked just as well as normal BAF in promoting a unified nucleus. Clearly, the mechanism by which BAF promotes a unified nucleus does not involve LEM-domain proteins.

One exciting possibility was that the DNA-binding capacity of BAF might drive 
the formation of a single nucleus. When BAF was first described, one key feature noted was its ability to bind and crosslink two segments of viral $\mathrm{DNA}^{7}$. BAF is not picky; it can bind any double-stranded $\mathrm{DNA}^{8}$. Intriguingly, this DNA-bridging capacity is built into the structure of BAF proteins, because they exist as pairs called dimers, with their DNA-binding sites located on opposite sides of the dimer ${ }^{8}$.

Samwer and colleagues hypothesized that BAF's ability to connect distant DNA segments might be relevant to its action in nuclear assembly. To test this, they replaced normal BAF in human cells with a mutant version that was deficient in the ability to form dimers. When cells that contained this mutant divided, the authors observed the formation of micronuclei. This indicated that DNA bridging by BAF dimers is needed to collect chromosomes together in one nucleus.

The author's calculations suggested that BAF dimers are spaced about 49 nanometres apart on the chromosomal surface, and might form a network of BAF-DNA bridges that prevent nuclear-envelope membranes (which are approximately $60-100 \mathrm{~nm}$ in diameter ${ }^{9}$ ) from infiltrating between neighbouring chromosomes. Previous analysis ${ }^{10}$ of high-resolution images captured using electron microscopy provided another clue - the addition of BAF compresses the outermost layer of chromosomal DNA, giving it the appearance of a dense shell surrounding less-dense DNA. Samwer and colleagues showed that a dimerizationdefective version of BAF failed to compress chromosomes.

Intrigued by the possibility that BAF alters the physical properties of chromosomes, Samwer and colleagues placed purified human chromosomes on glass slides, with or without the addition of BAF, and carried out two key experiments. First, using a tiny cantilevered metal rod to poke the chromosomal surface, they discovered that BAF stiffens chromosomes (Fig. 1). Second, they added fluorescent molecules called dextrans, which had average diameters of either 4 or $49 \mathrm{~nm}$. Using microscopy, the team was able to observe the smaller dextrans infiltrating the internal chromosomal space, whereas the larger dextrans could enter this space only in the absence of BAF. This exclusion of larger molecules correlated with the authors' estimate that BAF dimers are spaced approximately $49 \mathrm{~nm}$ apart.

These results might open additional avenues of investigation into the molecular nature of the BAF-dependent chromosomal 'shield' and the biomechanics of nuclear assembly. Many questions remain to be addressed. Do BAF dimers function alone ${ }^{11}$, or do they selfassociate or bind to DNA-binding histone proteins ${ }^{12,13}$ to increase the degree of chromosomal compression? Do they recruit other proteins involved in capturing chromosomes? Furthermore, how do the BAF dimers preferentially coat the outermost surfaces of chromosomes and avoid being trapped on interior chromosomal surfaces?

It will be interesting to learn how BAF 'shields' are dismantled, and how BAF contributes to chromosome re-engagement with lamins and nuclear-membrane proteins ${ }^{6,14}$, because these molecular connections are essential for customizing $3 \mathrm{D}$ genome organization in specific tissues and organs ${ }^{15}$. Further research might move beyond cell division, perhaps investigating the possibility of targeting BAF for anticancer therapies ${ }^{16,17}$, or detailing the mechanisms whereby BAF influences genome integrity, gene regulation and virus infection. Future research might also investigate the proposed involvement of BAF in progeria, a human genetic condition associated with characteristics of premature ageing ${ }^{18}$.

Tejas Dharmaraj and Katherine L. Wilson are in the Department of Cell Biology, Johns Hopkins University School of Medicine, Baltimore, Maryland 21205, USA. e-mail:klwilson@jhmi.edu
1. Samwer, M. et al. Cell 170, 956-972 (2017).

2. Hatch, E. M., Fischer, A. H., Deerinck, T. J. \& Hetzer, M. W. Cell 154, 47-60 (2013)

3. Zhang, C.-Z. et al. Nature 522, 179-184 (2015).

4. Gekara, N. O. J. Cell Biol. 216, 2999-3001 (2017).

5. Margalit, A., Segura-Totten, M., Gruenbaum, Y. \& Wilson, K. L. Proc. Natl Acad. Sci. USA 102, 3290-3295 (2005).

6. Gorjánácz, M. et al. EMBO J. 26, 132-143 (2007).

7. Lee, M. S. \& Craigie, R. Proc. Natl Acad. Sci. USA 95 1528-1533 (1998).

8. Umland, T. C., Wei, S.-Q., Craigie, R. \& Davies, D. R Biochemistry 39, 9130-9138 (2000)

9. Shibata, Y., Voeltz, G. K. \& Rapoport, T. A. Cell 126, 435-439 (2006).

10.Segura-Totten, M., Kowalski, A. K., Craigie, R. \& Wilson, K. L. J. Cell Biol. 158, 475-485 (2002).

11.Bradley, C. M., Ronning, D. R., Ghirlando, R., Craigie, R. \& Dyda, F. Nature Struct. Mol. Biol. 12, 935-936 (2005).

12.Zheng, R., Ghirlando, R., Lee, M. S., Mizuuchi, K., Krause, M. \& Craigie, R. Proc. Natl Acad. Sci. USA 97, 8997-9002 (2000)

13. Montes de Oca, R., Lee, K. K. \& Wilson, K. L. J. Biol. Chem. 280, 42252-42262 (2005).

14. Haraguchi, T. et al. J. Cell Sci. 121, 2540-2554 (2008)

15.Wong, X., Luperchio, T. R. \& Reddy, K. L. Curr. Opin. Cell Biol. 28, 105-120 (2014)

16. Kim, S. H. et al. J. Pharmacol. Exp. Ther. 352 , 175-184 (2015).

17.Gorjánácz, M. Nucleus 5, 47-55 (2014).

18.Jamin, A. \& Wiebe, M. S. Curr. Opin. Cell Biol. 34, 61-68 (2015)

\section{Large quantum systems tamed}

\section{Quantum-computing devices can be more powerful than their classical counterparts, but controlling large quantum systems is difficult. Two studies report work that overcomes this challenge. SEE ARTICLE P.579 \& LETTER P.601}

\section{CHRISTINE MUSCHIK}

$\mathrm{P}$ redicting the behaviour of more than a few quantum particles is tricky. The problem is so difficult that, in general, it cannot be tackled using classical (nonquantum) computers, and this has motivated the quest to build quantum simulators controlled quantum devices that provide us with answers to questions about the nature of quantum matter. Quantum simulators can address fundamental problems in physics, ranging from exotic quantum phases to open questions in high-energy physics. On the more applied side, they might even help chemists to create low-cost fertilizers and organic batteries (see go.nature.com/2jvwchw). In the long run, they could revolutionize our ability to design materials and drugs ${ }^{1}$. Today, however, quantum simulators are still at an early stage of development. On pages 579 and 601, respectively, Bernien et al. ${ }^{2}$ and Zhang et al. ${ }^{3}$ report advances in this exciting endeavour.

We are only beginning to understand how to build quantum simulators. One method is to use digital simulations ${ }^{4}$, in which a sequence of logic operations is performed on a quantum computer. Another approach is to use analog simulations, in which a specific model is emulated. For example, a classical analog simulation was used to design the roof of Germany's Olympic Stadium in Munich, which consists of a tantalizing structure of membranes. To find such lightweight yet stable configurations, architect Frei Otto experimented with soap bubbles. The experiments of Bernien et al. and Zhang et al. are quantum versions of this scenario - the researchers used trapped particles instead of soap solution and studied quantum phase transitions rather than roof designs.

Bernien and colleagues trapped atoms using optical tweezers - laser fields that hold atoms in place. This technique has the advantage that large arrays of atoms with arbitrary patterns can be prepared quickly and deterministically. The authors used additional lasers to excite atoms from the ground state to a Rydberg state, in which one of the atom's electrons is far away from the nucleus. Rydberg atoms have a large electric dipole moment and are coupled by 